\title{
Recuperación de sílice residual de columnas de cromatografía en la síntesis sol-gel de pigmentos
}

\author{
M.C. GRAÑANA, J.B. VICENT, M. LLUSAR, M.A. TENA Y G. MONRÓS \\ Dpto. de Química Inorgánica i Orgánica, Universitat Jaume I, 12080, Castellón
}

\begin{abstract}
En este trabajo se caracteriza una sílice residual de procesos de cromatografía de columna y se analiza su utilización como precursor en la síntesis de los tres pigmentos triaxiales del circón (amarillo de praseodimio, turquesa de vanadio y coral de hierro) utilizando tanto la vía sol-gel coloidal, la coprecipitación y el método cerámico. La sílice residual se muestra como un precursor muy efectivo en la obtención de los colores del circón tanto vía cerámica como a partir de geles. La alta temperatura así como los relativamente largos periodos de retención utilizados en la síntesis de los pigmentos cerámicos, permite una total combustión de los orgánicos adsorbidos sobre la sílice.
\end{abstract}

Palabras clave: gel de silice, absorción de orgánicos, pigmentos cerámicos, circón.

Recycling silica waste from support chromatography process in the synthesis sol-gel of ceramic pigments.

The use of a silica waste of support chromatography process as raw material in the synthesis of ceramic pigments based in zircon network (yellow of praseodymium, turkish blue of vanadium and pink koral of iron), is analized in this study. The synthesis has been carried out by ceramic method and also by colloidal sol-gel and coprecipitation routes. Silica waste becomes an effective precursor in the zircon ceramic stains. Likewise, the high temperature and long soaking time used in the synthesis ensure the whole combustion of adsorbed organics on the waste.

Key words: silica gel, adsorbed organics, ceramic pigment, zircon.

\section{INTRODUCCIÓN}

La silica gel, se obtiene por procesos de deshidratación de silicatos alcalinos solubles que pueden generar sílice de tamaño coloidal conocidos comercialmente como LUDOX cuando el coloide es monodisperso y con tamaños en torno a los 30 nm, o SYTON cuando el material es polidisperso (1). El gel obtenido por desecación es tamizado con el fin de controlar la distribución granulométrica del particulado.

Tras la utilización como adsorbente selectivo en las columnas de cromatografía clásicas, la sílice queda impregnada de los compuestos orgánicos del eluyente (dimetileter, diclorometano, acetato de etilo, metanol, ciclohexano...) y de los compuestos cromatografiados en menor proporción.

Los pigmentos cerámicos basados en la red del circón $\left(\mathrm{ZrSiO}_{4}\right)$ vienen siendo objeto de investigación por ser ampliamente utilizados en la decoración cerámica por su estabilidad térmica en el horno y frente a la agresión de los vidriados. La serie de pigmentos del circón se inició con el turquesa de vanadio (Seabright 1947) (2), siguió con el amarillo de praseodimio (Seabright 1956) (3) y alcanza la tricromía con el coral de hierro (Seabright 1965) (4).

En todos estos sistemas colorantes, la utilización de precursores de alta reactividad es de gran interés industrial, con el fin de disminuir la temperatura de síntesis del pigmento (ahorro energético), así como para minimizar el número y proporción de mineralizadores (fluoruros, nitratos, cloruros) que son necesarios añadir en la formulación para obtener un buen rendimiento de color y que tienen una importante incidencia medioambiental en emisión.
Desde este punto de vista, la utilización de la sílice residual de cromatografía, puede ser una fuente de sílice altamente reactiva que permita alcanzar los objetivos medioambientales anteriores. Los orgánicos adsorbidos sobre la sílice pueden ser lavados o bien, dadas las condiciones con que transcurre la síntesis del pigmento (alta temperatura, condiciones oxidantes y relativamente largos tiempos de retención), es previsible una perfecta combustión de estos compuestos.

El objetivo de este trabajo es caracterizar una sílice residual de procesos de cromatografía de columna y analizar el efecto de su utilización como precursor en la síntesis de los tres pigmentos triaxiales del circón anteriormente mencionados.

\section{CARACTERIZACIÓN DEL RESIDUO}

La sílice residual cromatográfica fue sumistrada por los laboratorios de Química Orgánica de la Universitat Jaume I de Castellón. La sílice original procedía de CU Chemie Uetikon AG (Suiza), con una granulometría controlada por tamizado entre 60-200 $\mu \mathrm{m}$. Después de ser utilizada en las columnas, la sílice era secada al aire y depositada en bidones de polietileno de alta densidad cerrados de 50 L. En la Figura 1 se presentan micrografías tanto de la sílice original como de la residual de la columna. No hay aparentes cambios en el material, sólo la presencia de particulado fino en el residuo y un aspecto más redondeado, con menos aristas de las partículas en el residuo, indican el efecto de la manipulación de la misma. El aspecto 


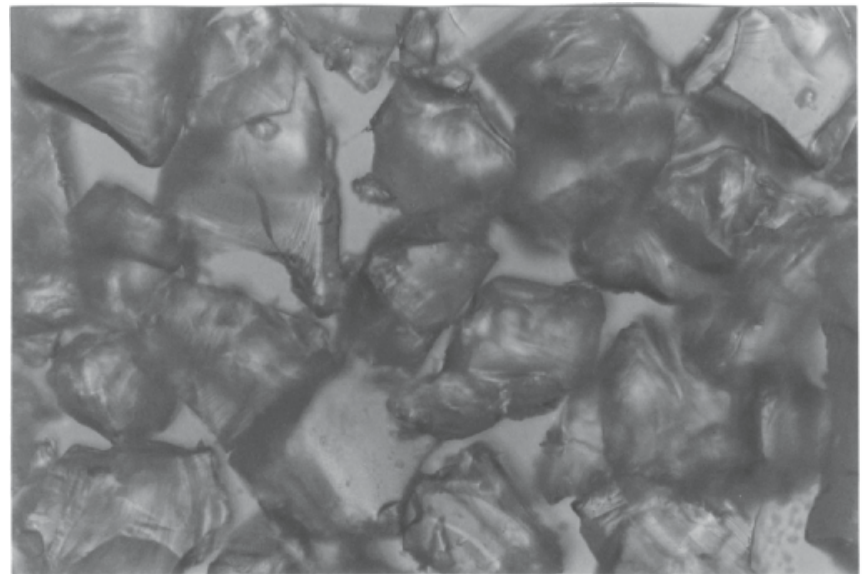

(a) sílice original

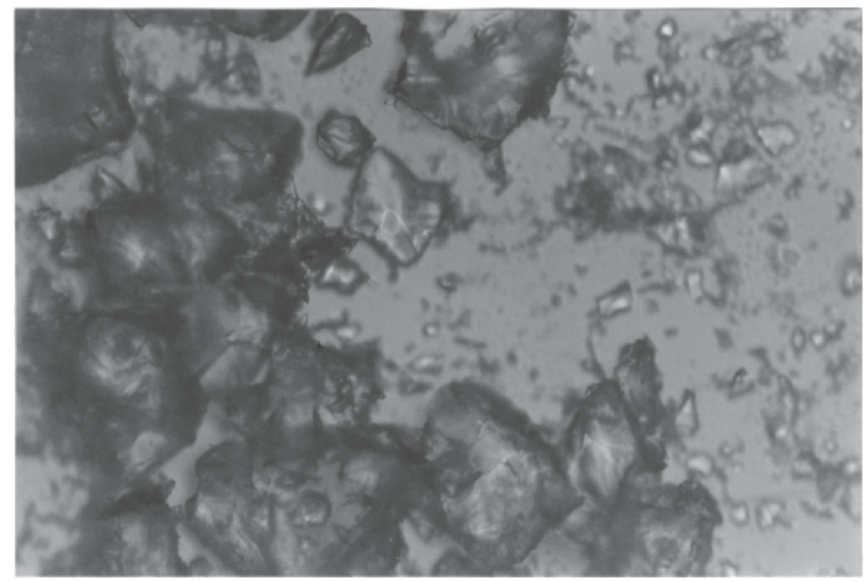

(b) residuo

Figura 1: Micrografías ópticas de la sílice original y la residual (x100).

anguloso de las partículas hacen referencia a su origen (la molturación de un gel seco monolítico), asimismo el tamaño observado de las partículas oscila entre los 100 y los $200 \mu \mathrm{m}$.

La sílice residual fue sometida a lavado con agua destilada con un $6 \%$ de agentes tensioactivos (5\% de sulfato de sodio dodecilo y $1 \%$ de alquilbencildimetilamonio). A $100 \mathrm{~g}$ de sílice residual se le añadió disolución lavadora hasta completar 500 $\mathrm{mL}$, la suspensión se homogeneizó en molino a $800 \mathrm{rpm}$ durante 20 minutos añadiendo $100 \mathrm{~g}$ de bolas de alúmina de dos tamaños (150 y $80 \mathrm{~mm}$ de diàmetro aproximadamente).
Después de la homogeneización, se separó el agua de lavado por decantación. La operación se realizó tres veces consecutivas. La sílice lavada se secó en estufa a $110^{\circ} \mathrm{C}$.

La sílice residual y la lavada fueron estudiadas mediante las siguientes técnicas: análisis térmico calorimétrico y gravimétrico simultáneos en un equipo Mettler (ATD-TG) a $1000^{\circ} \mathrm{C}$ con subida de $10^{\circ} \mathrm{C} / \mathrm{min}$, espectroscopia IR mediante el método convencional de la pastilla de $\mathrm{KBr}$ en un instrumento PerkinElmer así como por difracción de rayos $\mathrm{X}$ en un equipo Siemens D500.

En la Figura 2 se presentan las curvas ATD-TG de la sílice residual y lavada. De la Fig 2 se desprende que en el lavado se han eliminado gran parte de los orgánicos adsorbidos: la pérdida en la calcinación baja del $8 \%$ en el residuo al 3,5\% en la muestra lavada, por otro lado las bandas de ebullición (75 y $170^{\circ} \mathrm{C}$ ) desaparecen y la de combustión en $300-400^{\circ} \mathrm{C}$ mantienen la intensidad. Aparentemente los orgánicos no volátiles no son eliminables por lavado.

En la Figura 3 se presentan los difractogramas de rayos $X$ tanto del residuo (Fig.3-a) como de la muestra lavada (Fig.3-b), así como del material residual tras el tratamiento ATD-TG en las dos muestras. Ambas muestras crudas presentan un difractograma similar con un halo de desvitrificación centrado en $22^{\circ} 2 \theta$. Sin embargo los materiales residuales de ATD-TG presentan un comportamiento muy distinto: mientras que el residuo cristaliza cuarzo en forma moderada y manteniendo el halo a $22^{\circ} 2 \theta$, la muestra lavada presenta una fuerte cristalización de cristobalita y ha desaparecido el halo.

En la Figura 4 se presentan los espectros IR de ambas muestras. Las dos muestras presentan las bandas asociables al retículo de sílice ${ }^{[5]}$ : las bandas de tensión asimétrica Si-O-Si a 1200 y $1060 \mathrm{~cm}^{-1}$ que aparecen intensas en ambos materiales, la banda de tensión simétrica a $800 \mathrm{~cm}^{-1}$ muy intensa en las dos muestras, la banda de flexión a $470 \mathrm{~cm}^{-1}$ también intensa en las dos y la banda a $960 \mathrm{~cm}^{-1}$ de intensidad media en el residuo y débil en la muestra lavada asociable a la tensión del enlace $\mathrm{Si}^{-} \mathrm{O}^{-}$donde $\mathrm{O}^{-}$indica un oxígeno que no hace puente con otro átomo. Por otro lado se observa una banda a $600 \mathrm{~cm}^{-1}$ que pierde intensidad en la muestra lavada, puede asignarse a cloro en forma de cloruros o de enlace $\mathrm{Cl}-\mathrm{C}$, a $1.400 \mathrm{~cm}^{-1}$ se detecta otra banda de débil intensidad asociable a tensiones $\mathrm{H}-\mathrm{C}-\mathrm{H}$, a 3.500 y $1.635 \mathrm{~cm}^{-1}$ se detectan bandas intensas asociables a agua molecular y tensión del enlace $\mathrm{O}-\mathrm{H}$ respectivamente. La banda a $1.717 \mathrm{~cm}^{-1}$ de intensidad media en el residuo asociable a tensión del enlace $\mathrm{C}=\mathrm{O}$ disminuye ostensiblemente de intensidad en la muestra lavada, por último aparecen tres
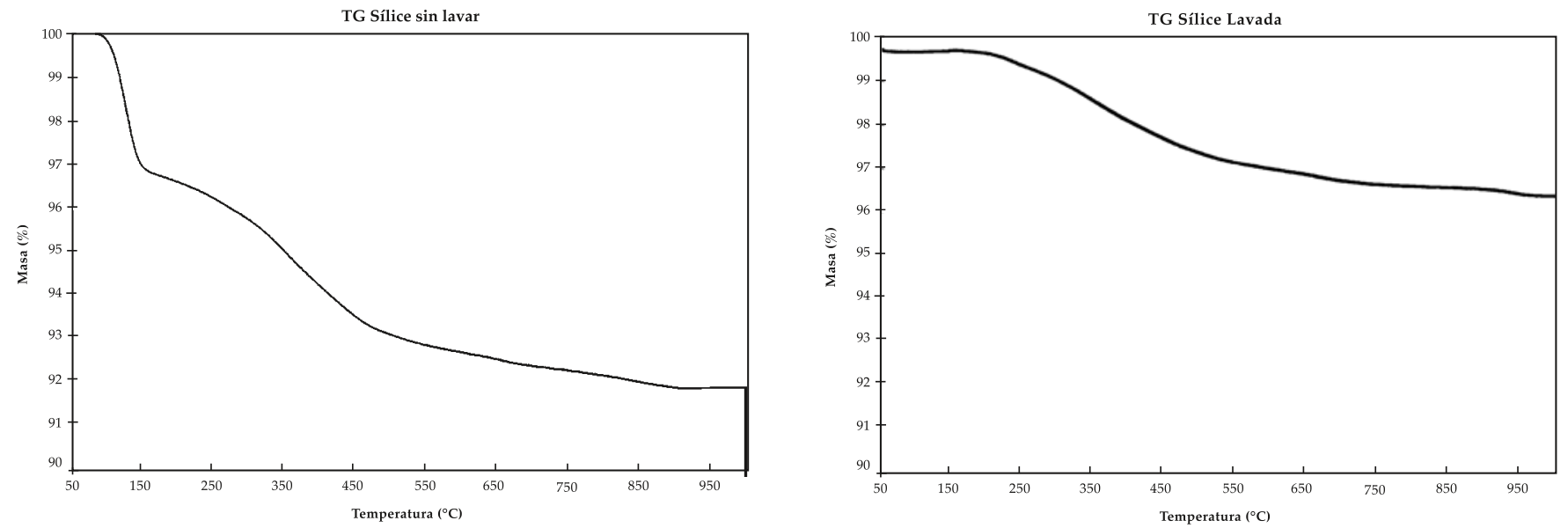

Figura 2: Curvas ATD-TG de la sílice residual y lavada. 
bandas asociadas en el residuo a 3.200 (intensa), 2.900 (débil) y $2.800 \mathrm{~cm}^{-1}$ (débil) que podrían relacionarse con enlaces $\mathrm{C}=\mathrm{O}$ y $\mathrm{C}-\mathrm{O}-\mathrm{C}$, estas bandas aparecen mejor resueltas en la muestra lavada. En definitiva los espectros IR ponen de manifiesto la pérdida de compuestos orgánicos en el material, si bien la retención tras el lavado sigue siendo importante. Asimismo la disminución de la banda de $800 \mathrm{~cm}^{-1}$ así como la mejor resolución de las bandas a 3.200 (intensa), 2.900 (débil) y $2.800 \mathrm{~cm}^{-1}$ indicaría la desadsorción de orgánicos desde el reticulado de sílice.

Desde el punto de vista medioambiental el lavado no es capaz de eliminar los orgánicos adsorbidos de forma completa y la contaminación que su presencia supone se traslada ahora a las aguas de lavado. Por otro lado estos orgánicos quimioadsorbidos son activos en la calcina del material, induciendo la cristalización de cuarzo en lugar de la cristobalita.

En general la metaestabilización de cristobalita (6), indica una mayor homogeneidad del sistema. Por tanto cristaliza vía nucleación homogénea, siempre más difícil y con fácil metaestabilización o formación de vidrios. Ya que en la síntesis de pigmentos cerámicos, con el objetivo de obtener a baja temperatura y con buen desarrollo la estructura huésped, es importante que el sistema cristalice de forma simple, se ha elegido como precursor en las diferentes síntesis el residuo sin lavar.

Desde el punto de vista medioambiental, la utilización de la sílice sin lavar, dado que los tratamientos térmicos en la síntesis de los pigmentos son a relativamente alta temperatura y con periodos de retención suficientemente largos, la contaminación orgánica puede ser eliminada íntegramente y de forma limpia por la combustión completa desarrollada en el horno.

\section{UTILIZACIÓN DE LA SÍLICE RESIDUAL EN LA PREPARACIÓN DE LOS PIGMENTOS DEL CIRCÓN.}

Se ha estudiado la sílice residual anteriormente caracterizada como precursor de silicio en la preparación de los tres pigmentos cerámicos del circón mediante dos rutas de síntesis:

\section{(I) Método cerámico (CE).}

Estas muestras se obtienen por mezclado en molino de bolas de los precursores elegidos en cada caso, incluidos los mineralizadores, utilizando acetona como medio dispersante. Se deja evaporar la acetona y se calcina según ciclo preestablecido.

\section{(II) Método de coprecipitación de hidróxidos o gel coloidal (GC).}

Se disuelven o dispersan en agua los diferentes precursores elegidos, incluidos los mineralizadores. A la disolución ácida resultante, mantenida a $70^{\circ} \mathrm{C}$ y en agitación continua, se añade disolución amoniacal hasta gelificación o pH 9 (en el caso de no formarse el gel). El gel o el coprecipitado obtenido se seca en estufa a $110^{\circ} \mathrm{C}$ y se calcina según el ciclo establecido.

De acuerdo con la literatura los tres pigmentos del circón presentan naturaleza muy diversa y ninguno se puede obtener por reacción directa entre el circón y las especies cromóforas. El azul de vanadio en circón es una disolución sólida de $\mathrm{V}^{4+} \mathrm{y} \mathrm{V}^{+5}$ en la red de circón ocupando tanto posiciones tetraédricas como octaédricas (7), el amarillo de praseodimio tiene diversas interpretaciones, aunque clásicamente se considera una disolución sólida de $\mathrm{Pr}^{4+}$ en circón, a partir de datos
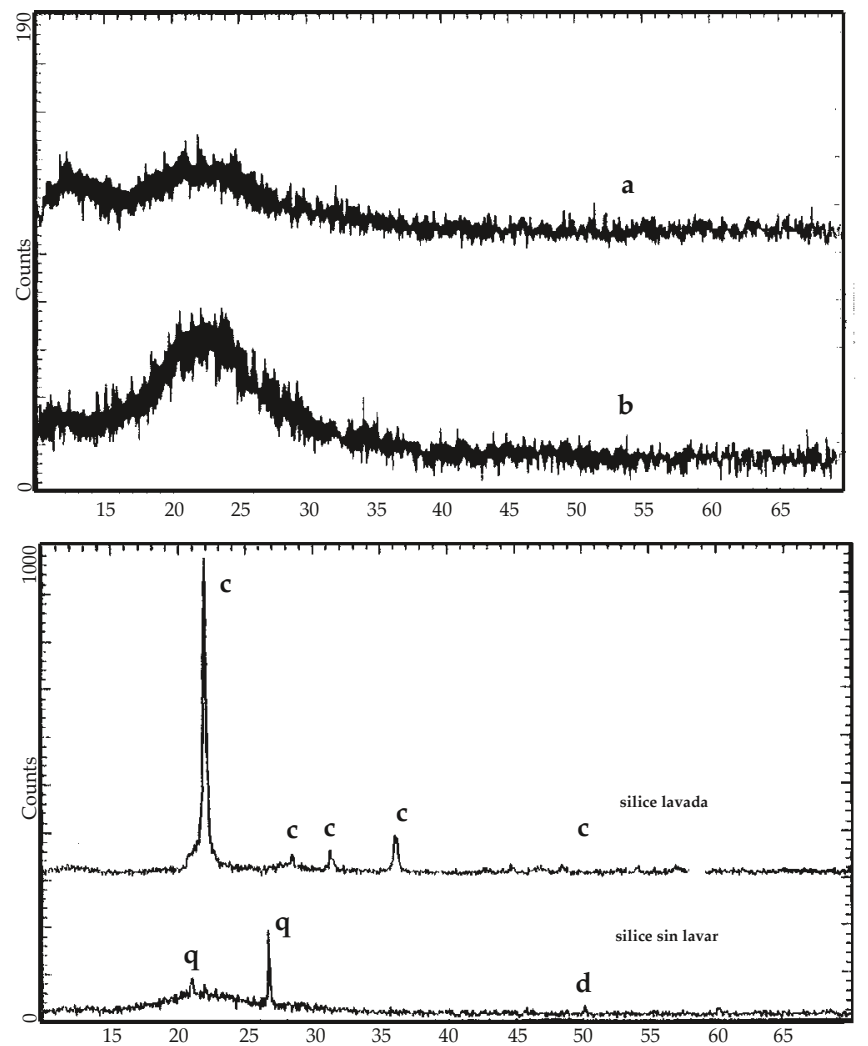

Figura 3: Difractogramas de rayos $\mathrm{X}$ del material residual: (a) sin calcinar y lavada, (b) sin calcinar y sin lavar, (c) lavada tras el tratamiento ATD-TG, (d) sin lavar tras el tratamiento ATD-TG . FASES CRISTALINAS: C(Cristobalita), (q) cuarzo.

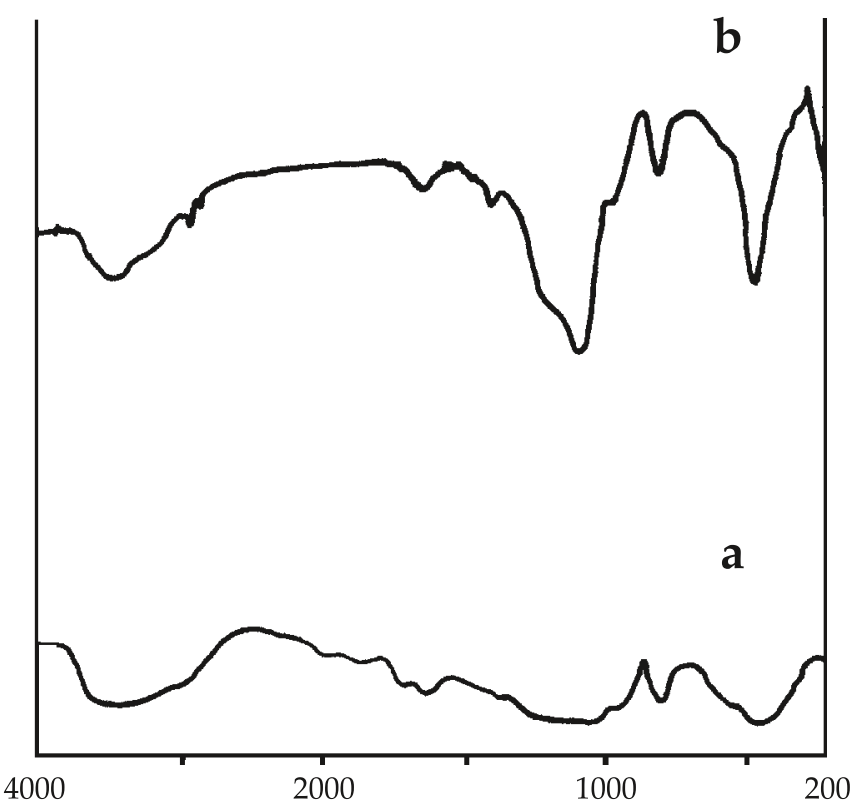

Figura 4: Espectros IR de ambas muestras: (a) sin lavar, (b) lavada. 


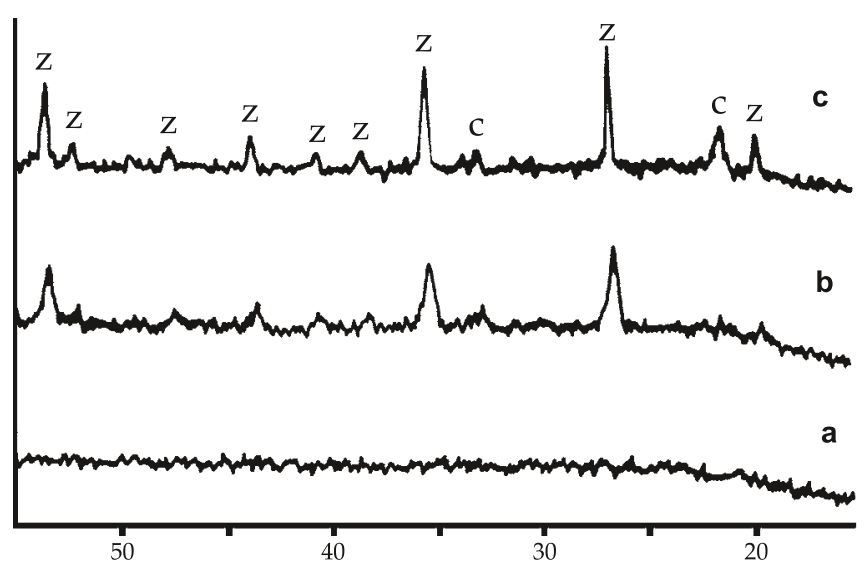

Figura 5: Evolución de la difracción de rayos $\mathrm{X}$ con la temperatura en la muestra 2-Fe: (a) muestra cruda, (b) $800^{\circ} \mathrm{C}$, (c) $1.000^{\circ} \mathrm{C}$. Fases cristalinas $=\mathrm{z}$ (circón) , c (cristobalita).

espectroscópicos y de reacción, se puede sugerir el encapsulamiento de un óxido de $\operatorname{Pr}^{3+}$ en circón, probablemente se den ambos mecanismos simultáneamente ${ }^{[8,9]}$. Por último, el rosa de hierro en circón es el resultado de hematita atrapada en cristales de circón (10).

Se han preparado los tres pigmentos a partir de los precursores y métodos presentados en la Tabla I: el turquesa de vanadio en circón (muestras $1-\mathrm{V}$ y $2-\mathrm{V}$ ) se han preparado por el método cerámico sustituyendo en la muestra 1-V el cuarzo por la sílice residual, en el amarillo de praseodimio (muestras 1-Pr y 2-Pr) y el coral (1-Fe y 2-Fe) se han preparado muestras cerámica y sol-gel utilizando en ambas la sílice residual.

Las muestras fueron calcinadas en horno eléctrico y crisoles silicoaluminosos a temperaturas sucesivas de 600, $800 \mathrm{y}$ $1.000^{\circ} \mathrm{C}$ según los casos, con tiempos de retención a la temperatura máxima de 6 horas.
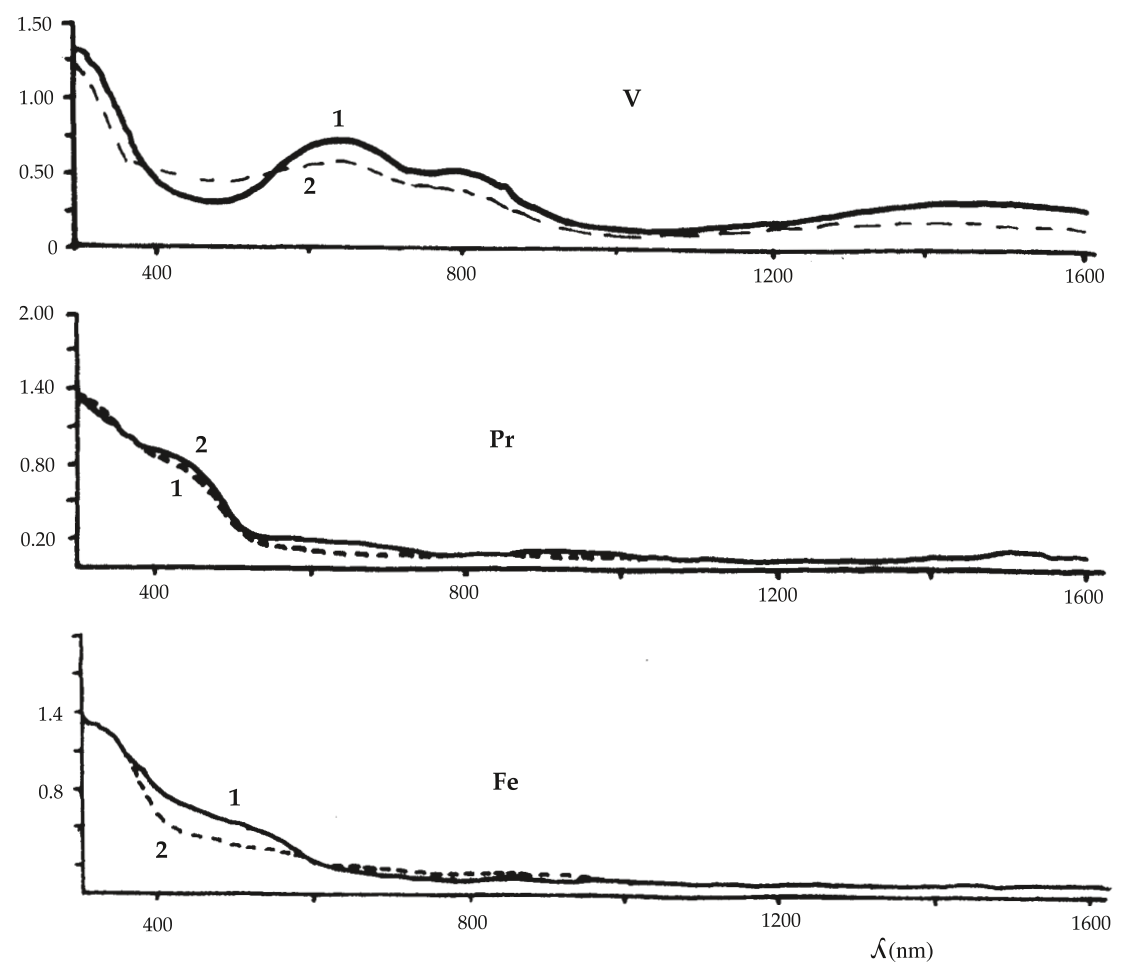

Figura 6: Espectros UV-V de las muestras lavadas calcinadas a $1000^{\circ} \mathrm{C}$.
Después de cada calcina las muestras se caracterizaron por Difracción de rayos $X$ para conocer la evolución tèrmica de las fases cristalinas. La muestra calcinada a $1.000^{\circ} \mathrm{C}$ fue micronizada en molino de bolas (800 rpm, 20 minutos), agregando el mismo peso de bolas (de dos tamaños) y el $60 \%$ del peso del polvo seco de agua. El agua de micronizado se separó por decantación y el polvo resultante fue lavado con $10 \mathrm{~mL}$ de agua por cada gramo de polvo después de agitar vigorosamente con ayuda de un agitador magnético durante 20 minutos la mezcla.

En el polvo lavado se han medido los parámetros reticulares del circón mediante los programas POWCAL y LSQC (16) y ha sido caracterizado por microscopía electrónica de barrido en un instrumento LEIKA 440i dotado de EDX de Oxford.

Por último el polvo fue esmaltado al $5 \%$ en peso con una frita borosílicea trasparente que fue cocida en horno rápido a $1.000^{\circ} \mathrm{C}$ con ciclo de 52 minutos cold-to-cold. La placa esmaltada fue caracterizada por espectroscopia UV-V por el método de reflectancia difusa así como por medidas colorimétricas CIEL*a* $b^{*}$.

En la Tabla I se presentan los precursores utilizados en los tres sistemas así como los resultados obtenidos en DRX, medidas colorimétricas CIEL ${ }^{*} \mathrm{a}^{*} \mathrm{~b}^{*}\left(\mathrm{~L}^{*}\right.$ indica la evolución de la claridad, a* la evolución de tono verde(-) $\rightarrow$ rojo(+) y b* la evolución azul $(-) \rightarrow$ amarillo(+)) así como los resultados de las dimensiones del retículo elemental del circón.

En la Figura 5 se presenta como evolución representativa el cambio de los perfiles de difracción de rayos $X$ de la muestra 2-Fe.

En la Figura 6 se presentan los espectros UV-V de las placas esmaltadas al $4 \%$ de las diferentes muestras.

En la Figura 7 se presentan micrografías representativas de los colores obtenidos.

Los resultados obtenidos indican una serie de hechos y fenómenos ya conocidos y recogidos por la literatura y que permiten analizar el comportamiento de la sílice residual en el desarrollo de los colores:

(i) Reactividad relativa de los sistemas y efecto de automineralización de las especies cromóforas.

La reactividad del sistema es muy alta en el caso del turquesa, inferior en el coral y baja en el amarillo de praseodimio de forma que a $1000^{\circ} \mathrm{C}$, el turquesa presenta prácticamente circón como única fase cristalina, el coral de hierro presenta ostensibles fases secundarias no reaccionadas de hematita y circona monoclínica y el amarillo los picos de circón y circona son de intensidad similar. Este extremo se debe al efecto automineralizador del pentóxido de vanadio en el azul, un cierto efecto en este sentido del óxido de hierro y la nula efectividad automineralizadora del óxido de praseodimio $(7,12,13)$.

Así como en la muestra sin lavar del residuo de sílice cristalizaba cuarzo al calcinar, en las muestras de los colores siempre se detecta cristobalita como fase intermedia no reaccionada de sílice lo que concuerda con estudios previos que indican la estabilización de la cristobalita en estos sistemas incluso partiendo de cuarzo (6). En todos los geles aparece como fase de reacción intermedia la circona tetragonal de bajo tamaño de cristalito (11). 
TABLA I: PRECURSORES UTILIZADOS Y RESULTADOS OBTENIDOS,

\begin{tabular}{|c|c|c|c|c|c|c|}
\hline Muestra & $1 . \mathrm{V}$ & $2-\mathrm{V}$ & 1.Pr & $2-\overline{P r}$ & 1 1-Fe & $2 \cdot \mathrm{Fe}$ \\
\hline Composición & $\begin{array}{l}\mathrm{V}_{0,0,0,5} \mathrm{Z}_{\mathrm{T}_{0.95}} \mathrm{SiO}_{4} \\
(\mathrm{aaF})_{0,2}\end{array}$ & $\mathrm{v}_{0,0,0} \mathrm{Z}_{\mathrm{r}_{0,05} \mathrm{~S}_{5} \mathrm{SO}_{4}}$ & $\mathrm{Pr}_{0,098} \mathrm{Z}_{\mathrm{T}, 9,95} \mathrm{SiO}_{4}$ & 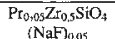 & 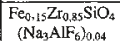 & 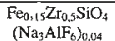 \\
\hline Método & $\mathrm{CB}$ & $C E$ & $\mathrm{CE}$ & $\mathrm{GC}$ & $\mathrm{CE}$ & $G C$ \\
\hline \\
\hline \multirow[t]{2}{*}{$\mathrm{Zr}$} & $\begin{array}{c}\mathrm{ZrO}_{2} \\
\text { monoclinica } \\
\text { SPR, }\end{array}$ & $\begin{array}{c}\mathrm{ZrO}_{2} \\
\text { monoclinica } \\
\text { SPR. }\end{array}$ & $\begin{array}{l}\mathrm{ZrO}_{2} \text { amorfa } \\
\text { SPR, } \\
\Phi_{s 0}=8,7 \mu \mathrm{m}\end{array}$ & $\begin{array}{c}\text { Acetato } \\
\text { Circonio } \\
\text { ALDRICH, }\end{array}$ & $\begin{array}{c}\mathrm{ZrO}_{2} \text { amorfa } \\
\mathrm{SPR}, \\
\phi_{50}=8.7 \mathrm{~mm}\end{array}$ & $\begin{array}{c}\text { Acetato } \\
\text { Circonio } \\
\text { ALDRICH. }\end{array}$ \\
\hline & $\begin{array}{l}\frac{\phi_{S \mathrm{Sl}}=3,9 \mu_{\mathrm{m}}}{} \\
\text { RESIDUO }\end{array}$ & $\begin{array}{c}\phi_{50}=3,9 \mu \mathrm{m} \\
\text { Cuarzo } 95,5 \%\end{array}$ & RESTIOOO & $\frac{22,4 \% \mathrm{en} \mathrm{ZrO}}{\mathrm{RESIDUO}}$ & RESIDUO & $\frac{22,4 \% \text { en } \mathrm{ZrO}_{2}}{\mathrm{RESIDUO}}$ \\
\hline Si & & $\begin{array}{c}\text { JJ NAVARRO, } \\
\phi_{50}=3,9 \mu \mathrm{m}\end{array}$ & & & & \\
\hline$v$ & $\mathrm{~V}_{2} \mathrm{O}_{5} 99 \%$ & $\mathrm{NH}_{4} \mathrm{VO}_{3} 99 \%$ & $\mathrm{Pr}_{6} \mathrm{O}_{11} 99 \%$ & $\mathrm{Pr}_{6} \mathrm{O}_{119} 99 \%$ & $\mathrm{FeSO}_{4} .7 \mathrm{H}_{2} \mathrm{O}$ & $\mathrm{FeSO}+7 \mathrm{H}_{2} \mathrm{O}$ \\
\hline \multirow[t]{2}{*}{$\mathrm{NaF}$} & $\begin{array}{l}\text { PANREAC } \\
\text { PANREAC }\end{array}$ & $\frac{\text { PANREAC }}{\text { PANREAC }}$ & $\frac{\text { FLUKA }}{\text { PANREAC }}$ & $\frac{\text { FLUKA }}{\text { PANREAC }}$ & \begin{tabular}{|l} 
PANREAC \\
INAVARRO
\end{tabular} & $\begin{array}{c}\text { PANREAC } \\
\text { J.NAVARRO }\end{array}$ \\
\hline & $\begin{array}{l}99 \% \\
\end{array}$ & $\begin{array}{l}99 \% \\
9\end{array}$ & $99 \%$ & $99 \%$ & $99 \%$ & $99 \%$ \\
\hline \multicolumn{7}{|l|}{$\begin{array}{c}\text { DRX } \\
\text { (color polvo) }\end{array}$} \\
\hline muestra cruda & A & $M(f) Q(f)$ & $\mathrm{PR}$ (mid) & $\mathrm{PR}(\mathrm{min})$ & $\mathrm{FeSO}_{4}, 7 \mathrm{H}_{2} \mathrm{O}(\mathrm{md})$ & A \\
\hline $600^{\circ} \mathrm{C} / 6 \mathrm{~b}$ & & $\mathrm{M}(\mathrm{t}) \mathrm{C}$ & 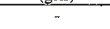 & & & \\
\hline $800^{\circ} \mathrm{C} / 6 \mathrm{~h}$ & $\overline{\mathrm{Z}(\mathrm{s}) \mathrm{M}(\mathrm{d})}$ & & $\overline{T(f M, Z(n)}$ & $\overline{\text { Z,TC(m)M(md) }}$ & $T(f) M(f)$ & $\mathrm{Z}(\mathrm{m}) \mathrm{H(d)}$ \\
\hline \multirow[t]{2}{*}{$1.000^{\circ} \mathrm{C} / 6 \mathrm{~h}$} & $z(\mathrm{~m}) \mathrm{M}(\mathrm{d})$ & $\begin{array}{c}\mathrm{Z}(\mathrm{mf}) \\
\text { (rof }\end{array}$ & $\mathrm{M}(\mathrm{f}) \mathrm{T}, \mathrm{Z}$ (m) & $\begin{array}{l}\mathrm{Z,T}(\mathrm{T}) \mathrm{C}(\mathrm{m}) \mathrm{M}(\mathrm{d}) \\
\text { (amarillo) }\end{array}$ & $\mathrm{Z}(\mathrm{m}) \mathrm{M}(\mathrm{f}) \mathrm{T}, \mathrm{H}(\mathrm{d})$ & $\mathrm{Z(f)} H, C(\mathrm{~d})$ \\
\hline & $\frac{\text { (turguesa) }}{65 /-12 /-13}$ & $74 /-12 /-7$ & $92 /-5 /$ & $877-8 / 30$ & & $\frac{70 j 0 / 10 / 21}{761}$ \\
\hline \multicolumn{7}{|l|}{$\begin{array}{l}\text { P. Reticulares } \\
\left(1000^{\circ} \mathrm{C}\right)\end{array}$} \\
\hline 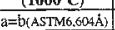 & $6,59(1)$ & $227(9)$ & & $6,619(9)$ & & \\
\hline & & & & $6,00(2)$ & & \\
\hline
\end{tabular}

(ii) El equilibrio de especies y sustituciones reticulares en el azul de vanadio en circón.

En el turquesa de vanadio, la evolución del color así como el tamaño de la red depende del grado de disolución sólida alcanzado (V por Zr o Si y F por O) y el equilibrio entre las especies cromóforas $\mathrm{V}^{4+}$ y $\mathrm{V}^{5+}$ (la entrada de $\mathrm{V}^{+5}$ incrementa los tonos verdes), así el material cerámico presenta coloraciones azules más intensas (ver espectros en Figura 6) y tamaños de celda inferiores que el circón sin dopar (ver valores ASTM en la Tabla I) que indican una mayor extensión en la entrada de flúor en el retículo $(7,9,10)$. En este sentido la utilización del residuo de sílice en la muestra cerámica $1-\mathrm{V}$ permite obtener mejores azules incluso utilizando un precursor clásicamente menos reactivo en este sistema como el $\mathrm{V}_{2} \mathrm{O}_{5}$ frente al metavanadato de la muestra $2-\mathrm{V}$.

En la Figura 4 se presenta el aspecto de la microestructura de la muestra $1-\mathrm{V}$ observándose particulados entre 3 y $5 \mu \mathrm{m}$ formando agregados de $15-20 \mu \mathrm{m}$.

(iii) Acuerdo de los datos con la naturaleza mixta del amarillo de praseodimio en circón.

En el amarillo de praseodimio, el color no parece asociarse con la reactividad del sistema, de forma que las muestras pierden coloración amarilla en el caso de la muestra GC desde 800 a $1000^{\circ} \mathrm{C}$, este hecho ya contrastado en la literatura $(8,12)$ se asocia con pequeñas variaciones expansivas de la red de circón evidenciando una naturaleza mixta del pigmento (disolución sólida y encapsulamiento de óxido de praseodimio). Los espectros UV-V del sistema de la Figura 6 indican Pr en el estado de oxidación +3 con una fuerte interacción en la zona ultravioleta-azul (17).

La utilización del residuo de sílice es óptima en ambos métodos. Como ya se describe en la litertura (9), la metodología sol-gel con coloides permite unos niveles de coloración amarilla a más baja temperatura así como una mayor reactividad del sistema.

La micrografía de la Figura 7 muestra una morfología irregular de los microcristales de circón con tamaños en torno a 1-6 $\mu \mathrm{m}$ en la muestra mejor coloreada 2-Pr.

(iv) Mantenimiento de las dimensiones de la celda elemental del circón en el coral de hierro y naturaleza encapsulada del pigmento.

En el caso del coral de hierro en circón, las dimensiones de la red no se modifican en el color, asimismo el color no se asocia
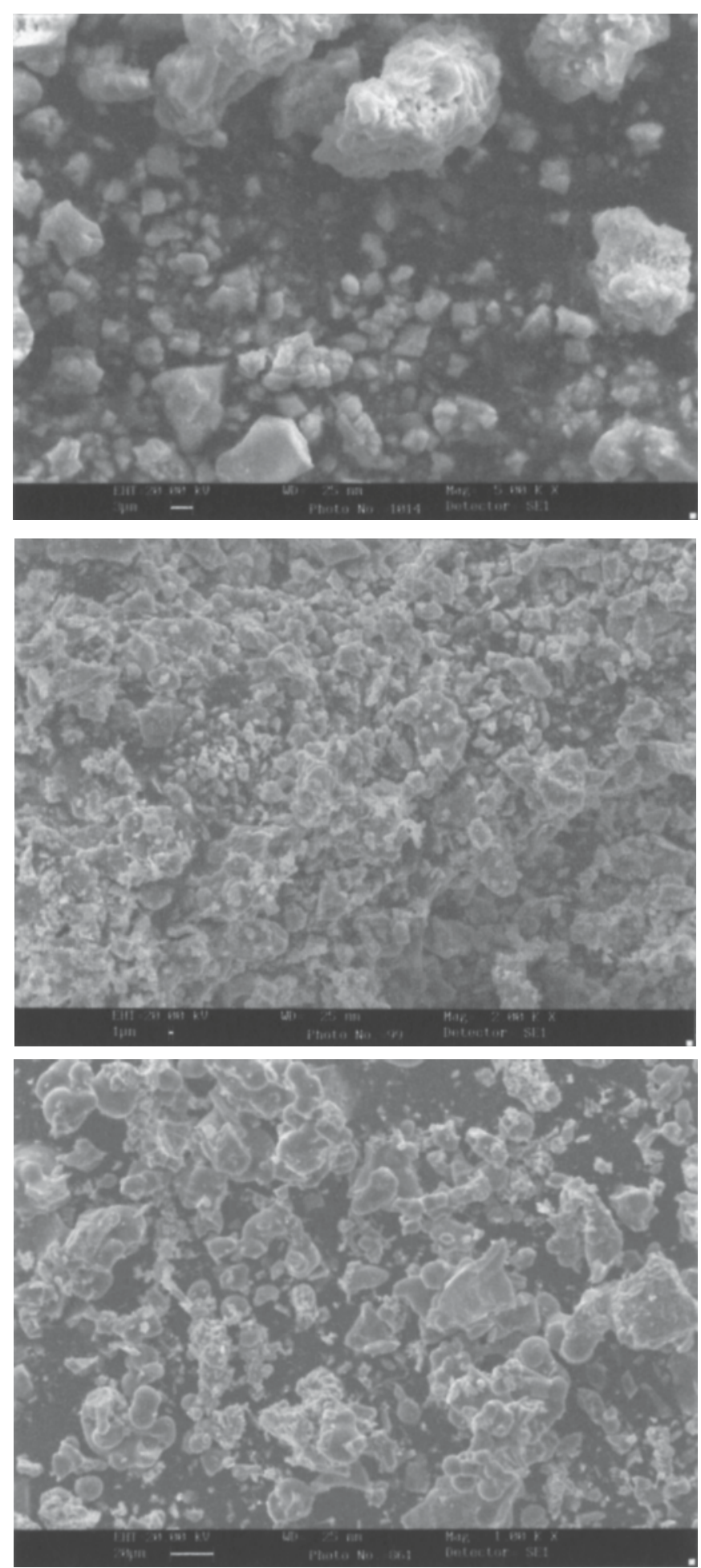

Figura 7: Micrografías electrónicas de los pigmentos: 1-V (arriba), 2-Pr (centro) y 1-Fe (abajo).

de forma directa con la reactividad del sistema: la muestra cerámica menos reaccionada y con una presencia importante de circona monoclínica presenta un nivel de color muy superior a la muestra gel con circón totalmente desarrollado. La necesidad de una sincronía en la cristalización de la hematita y la sinterización del circón descrita en la literatura $(13,14,15)$ explica bien estos fenómenos.

La utilización de la sílice residual permite obtener en la vía cerámica y con una mineralización muy simple unos niveles 
de coloración homologables a muestras con cuarzo con mineralización más compleja y más concentrada (NaF-NaCl o NaF$\mathrm{Na}_{2} \mathrm{AlF}_{6}$ de la literatura (13)).

En la Figura 7 la micrografía electrónica de la muestra de coloración rosa 1-Fe confirma un tamaño entre 4 y $8 \mu \mathrm{m}$ de las partículas, asimismo sobre las partículas de circón se observan partículas nanométricas del mineralizador condensadas tras su fusión en la calcina.

\section{CONCLUSIONES.}

1. El residuo de sílice de columnas cromatográficas es un producto amorfo de tamaño de partícula relativamente alto $(200 \mu \mathrm{m})$ con adsorciones de orgánicos del orden de un $7 \%$ en peso.

2. El principal problema medioambiental de este residuo sería la emisión a la atmósfera de los restos de disolvente eluyente durante su secado al aire que puede suponer hasta un $30 \%$ en peso del material. Esta emisión, de no estar correctamente ventilados los laboratorios, puede ser causa de graves problemas higiénico sanitarios en los mismos.

3. El lavado de la sílice para eliminar orgánicos, no resuelve el problema de los orgánicos adsorbidos (sólo se lavan el $50 \%$ de estos). Sin embargo, el lavado si que presenta efectos sobre el comportamiento en la calcina del material: el residuo sin lavar cristaliza cuarzo y el lavado cristobalita.

4. En la síntesis de los pigmentos cerámicos del circón se utilizan temperaturas y tiempos suficientes de retención como para asegurar una perfecta combustión de los orgánicos adsorbidos. La presencia de halógenos entre ellos no es un problema grave en estos sistemas industriales que utilizan mineralizadores halogenados y, por tanto, deben depurar sus emisiones al exterior.

5. Contrariamente a lo observado con el residuo simple, no se ha detectado nunca la cristalización de cuarzo al utilizarlo en la síntesis de los pigmentos del circón. El residuo siempre ha cristalizado, si lo ha hecho, cristobalita.

6. El residuo presenta un comportamiento notable en la síntesis de los pigmentos de la familia del circón (azul de vanadio en circón, amarillo de praseodimio-circón y coral de hierro-circón), tanto en la vía cerámica como en vías geles. Los resultados confirman los datos experimentales ya conocidos de los sistemas respecto de reactividad y desasrrollo colorimétrico.

\section{BIBLIOGRAFÍA}

1. Dupont Speciality Chemicals, LUDOX and SYTON: colloidal silica, Properties, Uses Storages and Handling, Publicación propia, Suiza, $10 / 1995$.

2. C.A. Seabright, Ceramic Pigments, U.S. Pat., 2(1948), 407-441

3. C.A. Seabright, US Pat. 2,992,123, July 11, 1961.

4. C. A. Seabright, Iron, Ceramic Pigment, U.S. Pat. 3166430,1965.

5. M. Nogami, Glass preparation on the $\mathrm{ZrO}_{2}-\mathrm{SiO}_{2}$ System by the Sol-Gel Process from Metal Alkoxides, J. Non Crystal. Sol., 69(1985),415-423.

6. G. Monrós et al., Synthesis and reactivity of cristobalite obtained from the $\mathrm{SiO}_{2}-\mathrm{V}_{2} \mathrm{O}_{5}$ system, J. of Mat. Sci. Lett. 9(1990),484-488.

7. G. Monrós et al., The Stoichiometry of Blue Vanadium Doped Zircon Obtained by Sol-Gel Methods", Mat. Res. Bull., 27(1992), 753-758.

8. M. Ocaña, A. Caballero, A.R. Gonzalez Elipe, P. Tartaj, C.J. Serna, Valence and localization of Praseodymium in Pr-Doped Zircon, J. of Sol. State. Chem, 27(1998) 412-415.

9. G. Monrós et al., Different Kinds of Solid Solutions in the $\mathrm{V}_{2} \mathrm{O}_{5}-\mathrm{ZrSiO}_{4}-\mathrm{NaF}$ System by Sol-Gel Processes and their Characterization, J. of Europ. Ceram. Soc. 11(1993) 77-86.

10. G. Monrós et al., Synthesis of $\mathrm{V}-\mathrm{ZrSiO}_{4}$ solid solutions, J. of Mat. Sci. Lett, 9(1990) 184-186

11. R.C. Garvie, Stabilization of the Tetragonal structure in Zirconia Microcrystals, J. Phys. Chem., 82(1978) 218-224.

12. J. Badenes et al., Análisis de las variables de síntesis del pigmento amarillo de praseodimio en circón, Bol. Soc. Cer. Vidr. 34(1995)147-152.

13. M. Llusar et al., Estudio del efecto de adición de distintos mineralizadores en la síntesis cerámica del pigmento rosa coral de hierro en circón, Boletín de la Sociedad Española de Cerámica y Vidrio, 38(1999),201-208.

14. P. Tartaj, Iron Zircon Pigments Pepared by Pyrolisis of Aerosols, J. of Sol. State Chem., 22(1997)102-108.

15. Chi-Hang Li, D.R. Eppler, R.A. Eppler, Iron zircon pigments, Ceram. Eng. Sci. Proc., 13(1992)109-118.

16. POWCAL y LSQC, Programas de cálculo, University of Aberdeen, Dpt. of Chemistry, U.K. 1990. 77-86.

17. G. DE. A. Licciuli, M. Nacucchi, “Uniformly dispersed $\operatorname{Pr}^{3+}$ doped silica glass by the sol-gel process", J. of Non-Crystalline Solids, 201(1996)153-158.

Recibido: 15-1-99

Aceptado: 28-12-99 